\title{
Analysis of prognostic significance of ventricular arrhythmias after myocardial infarction Shortcomings of Lown grading system
}

\author{
J THOMAS BIGGER JR, FRANCIS M WELD
}

From the Division of Cardiology, Department of Medicine, Columbia University; and The Cardiology Service, The Presbyterian Hospital in the City of New York

SUMMARY The Lown grading system for ventricular arrhythmias has been used in observational and experimental studies of ischaemic heart disease. This grading system uses three levels of ventricular premature depolarisation frequency and four complex features to assign patients to one of seven grades. We tested several of the major assumptions of the Lown grading system in a group of 400 patients who had recently experienced acute myocardial infarction. The Lown grading system assumes that the frequency of ventricular extrasystoles exerts a negligible risk force in patients who have complex ventricular extrasystoles. We found, however, that the frequency of ventricular extrasystoles contributed significant additional risk for cardiac death even in the three highest Lown grades, 4A, 4B, and 5 . The Lown grading system assumes that, of the four complex features used, $R$ on $T$ ventricular extrasystoles have the greatest risk for subsequent cardiac death. We found that paired ventricular extrasystoles and ventricular tachycardia had more prognostic significance than $R$ on $T$ ventricular extrasystoles.

It is important for prognostic stratification that subgroups which are merged into a given Lown grade should be relatively homogeneous with respect to outcome. We found a lack of homogeneity in the three highest Lown grades. Grade 5 contained 16 subgroups with a mortality risk which ranged from 0 to 75 per cent; statistically significant differences in subsequent mortality were found among these subgroups. Most of the shortcomings of the Lown grading system in our acute myocardial infarction population resulted from failure to give sufficient weight to ventricular extrasystoles frequency and to repetitive ventricular extrasystoles.

In 1971, Lown and Wolf proposed a grading system for ventricular arrhythmias encountered in ischaemic heart disease ${ }^{1}$; Lown and his colleagues have used the system up to the present time. The Lown grading system catalogues ventricular arrhythmias into seven ordered, mutually exclusive grades. Though Lown's 24-hour electrocardiographic recording may contain several complex features, the patient is assigned to the highest grade in the hierarchy. The hypothesis underlying this procedure is that patients in each grade in the hierarchy are at higher risk for subsequent cardiac death than those in lower grades. This grading system has been used for several small observational studies in ischaemic heart disease. ${ }^{2-4}$ In 1975, Lown et al. proposed an extension of the grading system, an "arrhythmia equation". 5 The arrhythmia equation enumerates

Received for publication 3 December 1980 the number of hours in each Lown grade and, in addition, provides some additional details about the arrhythmia in a 24-hour recording. ${ }^{5}$ The arrhythmia equation has not been adapted for use in observational studies. Lown and Graboys, however, have suggested the arrhythmia equation as an ideal means of judging the results of experimental studies, for example with antiarrhythmic drugs. ${ }^{6}$

The Lown system uses two ventricular extrasystoles frequency partition values and four complex ventricular extrasystoles features to grade arrhythmias. Table 1 gives the definitions for the Lown grades. The system is mutually exclusive and hierarchical. Grades are assigned on the basis of the highest ranking characteristic. For example, if $\mathbf{R}$ on $T$ is present, grade 5 is assigned regardless of ventricular extrasystoles frequency or other complex features. The three frequency categories $(0,>0$ but $<30,>30$ ventricular extrasystoles per hour) and 
Table 1 The Lown grading system for ventricular arrhythmias

\begin{tabular}{ll}
\hline Lown grade & Definition \\
\hline 0 & No ventricular premature depolarisations \\
1 & Less than 30 ventricular extrasystoles per hour \\
2 & 30 or more ventricular extrasystoles per hour \\
3 & Multiform ventricular extrasystoles \\
$4 \mathrm{~A}$ & Two consecutive ventricular extrasystoles \\
4B & Three or more consecutive ventricular \\
& extrasystoles \\
5 & R on T (RV/QT less than $1 \cdot 0)$ \\
\hline
\end{tabular}

four complex ventricular extrasystoles features give rise to 33 possible grades $\left(25_{+1}\right)$. The Lown grading system aggregates the 33 possible combinations of frequency and complex features into seven grades. The grades are assumed to be arranged in order of prognostic significance. For example, if ventricular tachycardia or $\mathbf{R}$ on $\mathrm{T}$ is present, these features are assumed to have such a potent mortality force that the presence of frequent ventricular extrasystoles or multiform ventricular extrasystoles is irrelevant. The purpose of our study was to test the assumptions of the Lown grading system in patients with definite ischaemic heart disease.

\section{Methods}

We evaluated the Lown grading system in a group of 400 patients who were treated for acute myocardial infarction at the Columbia-Presbyterian Medical Center. All patients had a diagnosis of acute myocardial infarction based on two of the following three criteria: (1) prolonged, substernal chest pain, (2) an increase in total creatine kinase to twice the normal level with a rise in the $M B$ fraction, (3) diagnostic $Q$ wave changes in the 12 lead electrocardiogram. All patients less than 70 years of age who were eligible and did not have other life-threatening disease were approached about the study. After discharge from the coronary care unit the study was explained to the patient and his physician. Informed, written consent was obtained between the sixth and tenth hospital day. A medical history, physical examination, and laboratory studies were performed on each patient after informed consent was obtained. Between 10 and 20 days after hospital admission, a 24-hour electrocardiographic recording was made using an Avionics two-channel recorder. A modified V1 and V5 lead were used in nearly all cases (96.2\%); other leads were used when necessary to obtain a QRS complex of adequate size. Patients were followed every six months by telephone contact and by questionnaires which were sent to the primary physicians. Deaths were investigated by interview- ing the next-of-kin, friends, and/or physician of each patient who died. Follow-up was available in 98 per cent of the patients who enrolled. During an average follow-up of 30 months, 78 deaths occurred in our population.

\section{ANALYSIS OF 24-HOUR \\ ELECTROCARDIOGRAMS}

Each 24-hour tape was analysed by a digital computer program, Columbia IV. ${ }^{7}$ The records were digitised at 250 samples per second. After a brief set-up by an operator, the computer analysed the tape. At the end of computer analysis, the record was edited by the operator to correct false positive and false negative ventricular premature depolarisations. Then, a statistical summary and electrocardiogram strips were made for review by one of the authors (JTB). Strips were made of the 20 shortest and longest RR and RV (last normal QRS to ventricular extrasystoles) intervals and 10 examples of each of the following were made: (1) each ventricular extrasystoles shape, (2) paired ventricular extrasystoles, (3) ventricular tachycardia, (4) ventricular bigeminy, (5) shortest $\mathrm{RV} / \mathrm{QT}$, (6) shortest RV/RR, and (7) shortest RV. The episodes of bigeminy and ventricular tachycardia were sorted in descending order so that the 10 longest episodes were always reviewed. If the cardiologist found errors in the analysis, the electrocardiogram and annotated QRS file were reloaded from magnetic tape and re-edited. After re-editing, an updated statistical summary and new set of electrocardiogram strips were produced for a second review by the cardiologist. Re-editing was required in about 15 per cent of the analyses.

\section{DEFINITIONS}

\section{Multiform ventricular extrasystoles}

During editing, the computer operator merged the ventricular extrasystoles shapes generated by Columbia IV into a smaller number. The operators were instructed to assign only four basic shapes in either lead according to the direction of the first and last portions of the QRS complex. The following four shape definitions were used: (1) entire QRS deflection positive; (2) entire QRS deflection negative; (3) initial QRS deflection positive, terminal deflection negative; (4) initial QRS deflection negative, terminal portion positive. Minor alterations in shape were ignored because these can be the result of changes in position, respiration, electrode-skin resistance, etc. Using this scheme, a maximum of four shapes is possible in either lead, and 16 shapes are possible in two leads. There were, however, usually five or less shapes in a 24-hour 
electrocardiographic recording even when ventricular extrasystoles were frequent.

Repetitive ventricular extrasystoles

Paired ventricular extrasystoles were defined as two consecutive ones regardless of the interval between them. Ventricular tachycardia was defined as three or more consecutive ventricular premature depolarisations, regardless of the $R R$ intervals. In only three of the 47 persons with ventricular tachycardia was the average $R R$ interval greater than $600 \mathrm{~ms}$ (that is, rate less than 100 a minute).

\section{$R$ on $T$ ventricular extrasystoles}

$\mathbf{R}$ on $\mathbf{T}$ was defined as a $\mathbf{R} \mathbf{R}^{\prime} / \mathrm{QT}$ interval less than $1 \cdot 0$. There is considerable technical difficulty in detecting the $R$ on $T$ phenomenon by computer or scanner and in assigning this characteristic by eye. We used three techniques in the attempt to detect $\mathbf{R}$ on $\mathbf{T}$ ventricular extrasystoles. During the computer run, we sorted and stored three intervals in ascending order: (1) $R V / \mathbf{R R}^{\prime}$ interval, (2) $R V / R R$ ratio, and (3) $R V / Q T$ ratio. The computer used the following procedure to estimate QT intervals. Before each tape was analysed, electrocardiographic strips were made at $50 \mathrm{~mm} / \mathrm{s}$. Care was taken to record a portion of the record which has a distinct $T$ wave. Five $R R$ and corresponding QT intervals were measured by a cardiologist and these values were typed into the computer by the operator during set-up for the computer analysis. From these $R R$ and $Q T$ intervals, a corrected QT (QTc) was computed. During computer analysis, $Q T c$ and $R R$ intervals were used to compute a QT interval for each QRS complex. During the post-edit review, the cardiologist reviewed electrocardiographic strips of the 10 shortest $R V, R V / R R$, and $R V / Q T$ intervals and decided whether $R$ on $T$ was present. In addition, he calculated the minimum RV/QT from the computer generated electrocardiographic strips.

Table 2 Relation between Lown grade and mortality in 400 patients after acute myocardial infarction

\begin{tabular}{lccc}
\hline Lown grade & Group total & \multicolumn{2}{l}{ Deaths } \\
\cline { 3 - 4 } & & No. & Per cent \\
\hline 0 & 64 & 9 & 14 \\
1 & 85 & 9 & 11 \\
2 & 2 & 0 & 0 \\
3 & 68 & 10 & 15 \\
4 A & 44 & 9 & 20 \\
4B & 21 & 7 & 33 \\
5 & 116 & 34 & 29 \\
All & 400 & 78 & 20 \\
\hline
\end{tabular}

STATISTICAL METHODS

Differences between ventricular extrasystoles characteristics or Lown grades with respect to outcome were evaluated using the $\mathrm{z}$ statistic for proportions or the $\chi^{2}$ statistic with the Yates correction. ${ }^{8}$ The critical value for declaring a difference was $\mathrm{p}<0.05$. Sensitivity, specificity, false positive rate, and false negative rate were computed by standard methods. 8

\section{Results}

HIERARCHY OF RISK

One of the assumptions of the Lown grading system is that an increasing gradient of mortality from low to high risk should exist through the hierarchy of Lown grades. Table 2 shows the number of deaths and the grade specific mortality rate for each Lown grade. The death rate does not steadily increase as a function of Lown arrhythmia grade. The mortality rates are approximately equal in the first five grades. The mortality rate is significantly higher in grades $4 \mathrm{~B}$ and 5 than in the other five grades $\left(\chi^{2}=13.4, \mathrm{p}<0.01\right)$. There was no

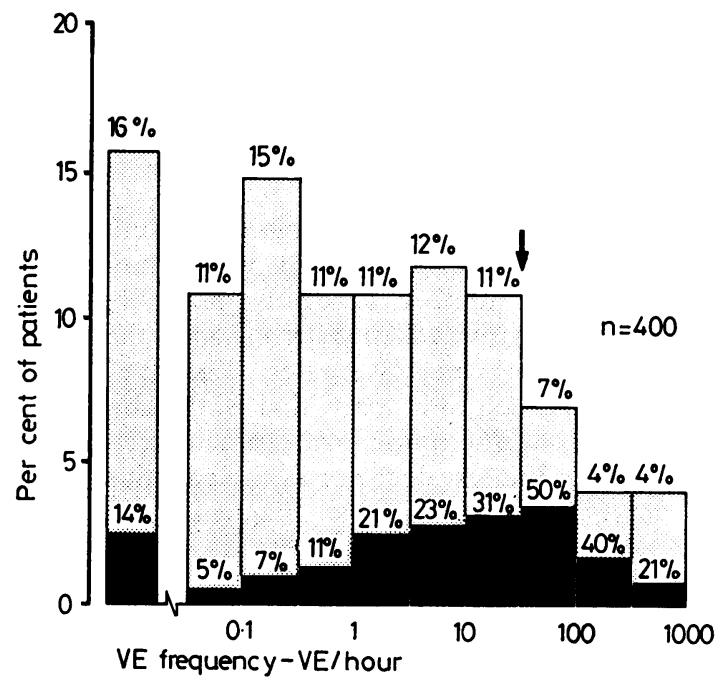

Fig. 1 Frequency histogram of ventricular extrasystoles frequency in 400 patients with recent acute myocardial infarction. The average 24-hour ventricular extrasystoles frequency is plotted on the $X$ axis on a log scale. The percentage of the total group in each half log interval is plotted on the $Y$ axis. The black, lower portion of each bar indicates the deaths in that category. The frequency specific mortality rates are indicated just above the black portion of the bar. The arrow indicates the partition value which separates Lown grade 1 from Lown grade 2 (30 ventricular extrasystoles ( $V E$ ) per hour). 
significant difference, however, between grade 4B and grade 5 . Thus, the Lown grading system performed poorly in terms of defining a gradient of risk. ${ }^{9}$

\section{FREQUENCY}

Fig. 1 shows the frequency distribution of ventricular extrasystoles in our group of 400 postinfarction patients and the relation of ventricular extrasystoles frequency to cardiac death. Several points are worthy of mention. First, the frequency distribution of ventricular extrasystoles frequency is extremely skewed in the positive direction. Hence, a log scale was used on the $\mathrm{X}$ axis. From a ventricular extrasystoles frequency of greater than 0 to 30, there is an almost equal proportion of the population in each half log unit. The Lown partition value of 30 per hour is indicated by the black arrow. In our patients, 30 ventricular extrasystoles per hour is the 86th centile, that is, only 56 patients $(14 \%)$ lie above this value. Second, over most of the ventricular extrasystole frequency range, the mortality rate increases as a function of frequency of ventricular extrasystole. From 0.03 to 100 ventricular extrasystoles per hour, the frequency specific mortality rate increases in each frequency interval. Departures from the increase in mortality, however, as a function of frequency of ventricular extrasystoles occur in both tails of the distribution. These departures could be the result of a sampling error, particularly at the upper end of the frequency distribution where the numbers are relatively small. Table 3 compares five possible partition values including the Lown criterion of 30 per hour with respect to the number of deaths detected, odds of dying, sensitivity, specificity, and the false positive and false negative rates. The five values are each a half log unit apart, that is, the same scale used in Fig. 1. The number of deaths detected and sensitivity of the test decrease as the partition value for ventricular extrasystoles frequency increases.

Table 3 Comparison of five ventricular extrasystoles frequency criteria for risk stratification in 400 patients after acute myocardial infarction

\begin{tabular}{lccccc}
\hline & \multicolumn{5}{c}{ Ventricular extrasystoles } \\
\cline { 2 - 6 } & $\geq 1$ & $\geq 3$ & $\geq 10$ & $\geq 30$ & $\geq 100$ \\
\cline { 2 - 6 } & 190 & 147 & 99 & 56 & 31 \\
No. of patients & 57 & 48 & 37 & 24 & 10 \\
No. of deaths & $23 \cdot 1$ & $24 \cdot 3$ & $23 \cdot 4$ & $17 \cdot 5$ & $2 \cdot 7$ \\
$\chi^{2}$ & $3 \cdot 7$ & $3 \cdot 6$ & $3 \cdot 6$ & $3 \cdot 6$ & $2 \cdot 2$ \\
Odds ratio & $73 \%$ & $62 \%$ & $47 \%$ & $30 \%$ & $13 \%$ \\
Sensitivity & $59 \%$ & $69 \%$ & $81 \%$ & $89 \%$ & $93 \%$ \\
Specificity & $71 \%$ & $67 \%$ & $64 \%$ & $60 \%$ & $68 \%$ \\
False positive rate & $10 \%$ & $12 \%$ & $14 \%$ & $16 \%$ & $18 \%$ \\
False negative rate & $10 \%$ & & & & \\
\hline
\end{tabular}

In addition, the specificity increases as the partition value increases. A judgement about the best partition value depends to a great extent on the application of the test results. ${ }^{10}$ If the low risk group is to be excluded from treatment and followed less often than other patients, one per hour is the best of these criteria: a very small proportion of the patients who will die or have new coronary events will be excluded from treatment or follow-up using this criterion. For treatments that carry a significant chance of morbidity or mortality, 30 per hour is a good criterion on which to choose the treatment: only about 15 per cent of the population is exposed to the risk of treatment and these patients already have a high likelihood of dying. For treatments which have low to moderate risk of adverse effects, the three or 10 per hour criterion may give the best combination of sensitivity and specificity.

Two additional points should be made about frequency of ventricular extrasystoles and the Lown grading system. First, the progressive rise in mortality rate with increasing ventricular extrasystoles suggests that the practice of using a single partition value of ventricular frequency to assign risk ignores much valuable information. Second, using Lown's grading system, frequency of ventricular extrasystoles will hardly ever be used to estimate the probability of risk when these extrasystoles are numerous. This latter fact stems from the very strong association between high frequency and complexity of ventricular extrasystoles in ischaemic heart disease patients and from the mutually exclusive Lown grades. In our population, 54 of the 56 patients $(96 \%)$ who had 30 or more ventricular extrasystoles per hour (eligible for Lown grade 2) moved to higher Lown grades because they also had one or more complex ventricular extrasystoles features. The associations among frequency of ventricular extrasystoles and the four complex features used in the Lown grading system in our patients are shown in Fig. 2. The phi coefficient is used to measure the strength of association. ${ }^{8} \mathrm{Phi}$ is interpretable in the same way as a product moment correlation coefficient; values less than 0.35 are taken to indicate insignificant associations. ${ }^{8}$ It can be seen that there are strong associations between high frequency of ventricular extrasystoles ( $>30$ per hour) and repetitive forms of ventricular extrasystoles.

One of the assumptions of the Lown grading system is that frequency makes a trivial contribution to mortality risk when complex features are present. We tested this assumption in several ways. In Fig. 3, we examine the relations among ventricular extrasystoles frequency, Lown grades 1 to 5 , and 


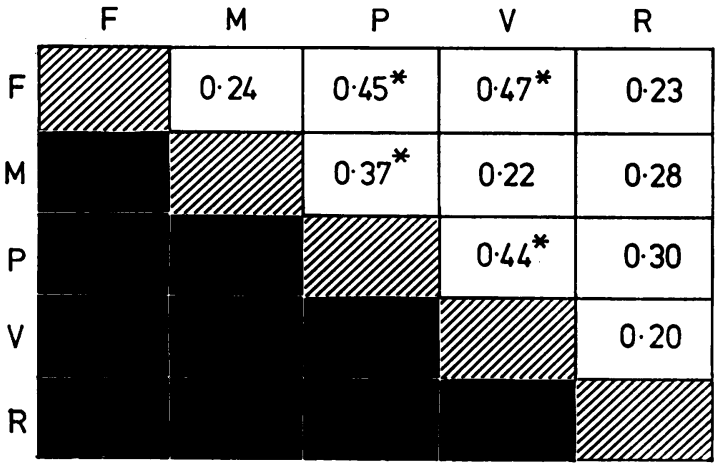

Fig. 2 The strength of association among the ventricular extrasystoles (VE) characteristics used in the Lown grading system. The associations are expressed as phi coefficients. ${ }^{8}$ The significant associations are indicated with asterisks. F, high frequency (that is $\geq 30$ per hour); $M$, multiform; $P$, paired ventricular extrasystoles; $V$, ventricular tachycardia; $R, R$ on $T V E$.

death. For each Lown grade, the height of the bar indicates the range of ventricular extrasystoles frequency observed in that grade. Except for grade 2 , which only contains two persons, the range of ventricular extrasystoles frequency extends over several orders of magnitude in each Lown grade. The median frequency is indicated by a horizontal line across each bar and deaths are indicated by

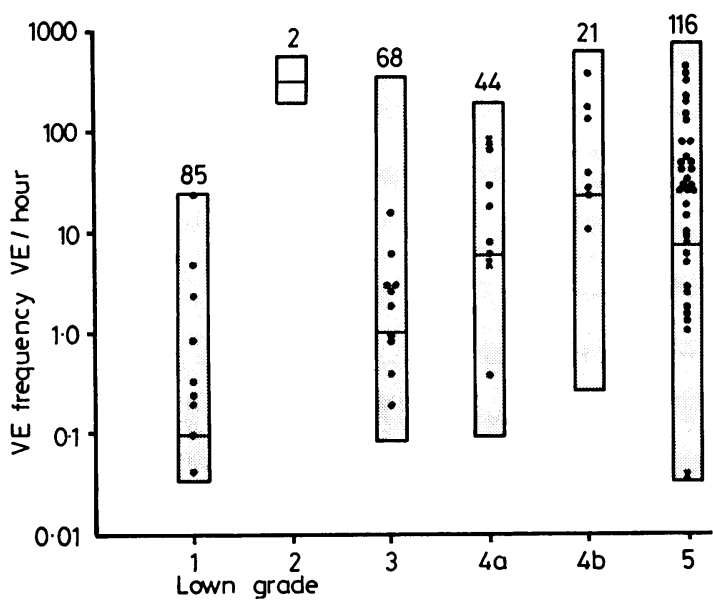

Fig. 3 The associations among Lown grade, ventricular extrasystoles frequency, and death. The numbers over each bar indicate the number of patients in each Lown grade. The horizontal line across each bar indicates the median ventricular extrasystoles frequency in each grade. In each Lown grade, most of the deaths are above the median ventricular extrasystoles frequency, indicating an association between high frequency and death.
Table 4 Contribution of frequency to mortality in Lown grades 3 to 5

\begin{tabular}{lccc}
\hline Lown grade & \multicolumn{3}{l}{ Average ventricular extrasystole frequency } \\
\cline { 2 - 4 } & $\geq 10 /$ hour & $<10 /$ hour & Total \\
\hline Grade 3 & $1 / 11(9 \%)$ & $9 / 57(16 \%)$ & $10 / 68(15 \%)$ \\
Grade 4A & $5 / 18(28 \%)$ & $3 / 26(12 \%)$ & $8 / 44(18 \%)$ \\
Grade 4B & $7 / 13(54 \%)$ & $0 / 8(0 \%)$ & $7 / 21(33 \%)$ \\
Grade 5 & $25 / 53(44 \%)$ & $9 / 63(14 \%)$ & $34 / 116(29 \%)$ \\
Total & $37 / 84(44 \%)$ & $12 / 97(12 \%)$ & $49 / 181(27 \%)$
\end{tabular}

Note: In each column, the denominator is the total number of patients in the category and the numerator gives the number of patients in the category who died. The percentage in parentheses is the mortality rate.

black dots. It can be seen that in every grade which has deaths (all but grade 2), the majority of the deaths are above the median frequency. This indicates that, in every Lown grade, ventricular

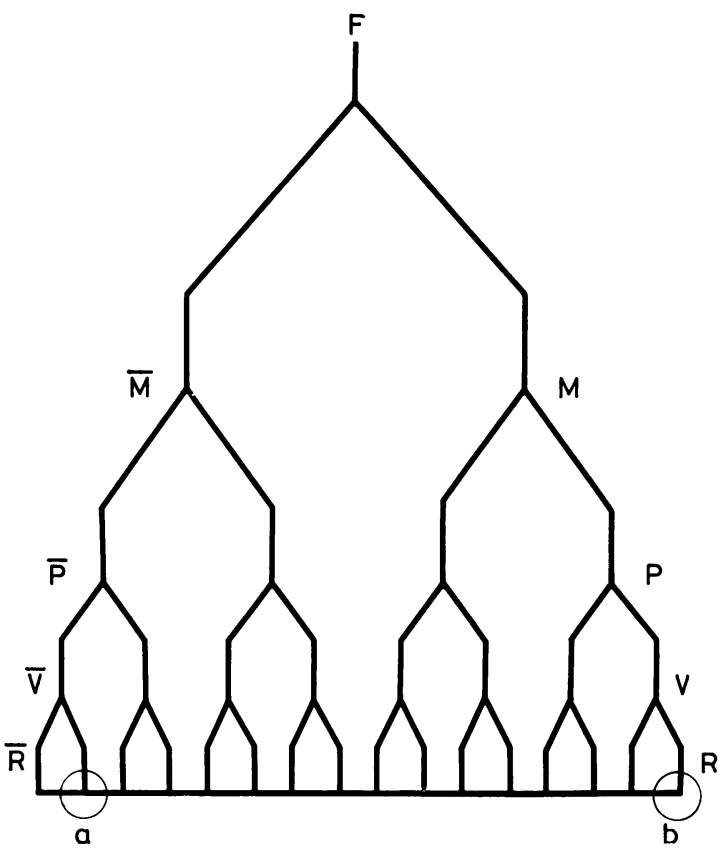

Fig. 4 Combinations of high frequency $(F)$, multiform ventricular extrasystoles $(M)$, paired ventricular extrasystoles $(P)$, ventricular tachycardia $(V)$, and $R$ on $T$ ventricular extrasystoles $(R)$. The bar over the letters on the left of the diagram indicates absence of the characteristic. A corresponding tree (not shown) exists for patients with low ventricular extrasystoles frequency (less than 30 per hour). Subgroup (a) at the bottom left of the diagram has a nil per cent mortality; subgroup $(b)$ at the bottom right of the diagram has $a$ 59 per cent mortality; subgroups (a) and (b) are both in Lown grade 5. 
Table 5 Heterogeneity of mortality rates in subgroups which comprise Lown grade 5

\begin{tabular}{|c|c|c|c|}
\hline \multirow[t]{2}{*}{ Subgroup } & \multirow[t]{2}{*}{ No. in group } & \multicolumn{2}{|c|}{ Died } \\
\hline & & No. & Per cent \\
\hline 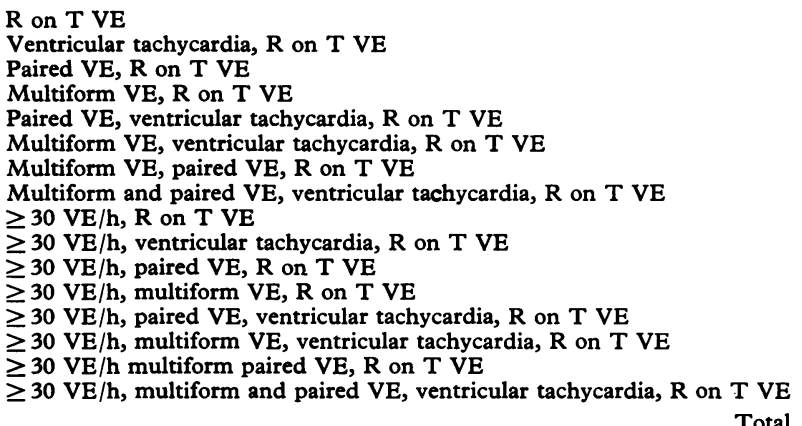 & $\begin{array}{r}22 \\
0 \\
4 \\
32 \\
1 \\
3 \\
18 \\
4 \\
1 \\
0 \\
3 \\
3 \\
1 \\
0 \\
7 \\
17 \\
116\end{array}$ & $\begin{array}{r}2 \\
0 \\
3 \\
3 \\
0 \\
1 \\
5 \\
3 \\
0 \\
0 \\
1 \\
1 \\
1 \\
0 \\
4 \\
10 \\
34\end{array}$ & $\begin{array}{r}9 \\
75 \\
9 \\
0 \\
33 \\
28 \\
75 \\
0 \\
33 \\
33 \\
100 \\
- \\
57 \\
59 \\
29\end{array}$ \\
\hline
\end{tabular}

extrasystoles frequency is exerting significant influence on mortality. In addition, we looked at the relations among frequency and complexity of ventricular extrasystoles and death in another way. Table 4 tests the hypothesis that frequency makes no significant contribution to risk in the presence of complex features. The high frequency criterion used is 10 per hour, a value that divides the population at the 75th centile. We tested whether or not high frequency of ventricular extrasystoles increased the probability of dying in persons who were in Lown grades determined by complex features, that is grades 3 to 5 . There is no difference between the low and high groups with respect to mortality in grade 3. However, in Lown grades $4 \mathrm{~A}, 4 \mathrm{~B}$, and 5 , the persons who had 10 or more ventricular extrasystoles per hour had a significantly higher mortality than persons in the same grade who had less than 10 ventricular extrasystoles per hour. This result clearly indicates that their frequency continues to exert its adverse influence even in persons who have complex ventricular extrasystoles features.

\section{COMPLEX FEATURES}

Grades 3 to 5 of the Lown system represent aggregates of 30 distinct subgroups. Fig. 4 diagrammatically shows the combinations of high frequency and the complex features used in the Lown grading system; a corresponding tree exists for combinations of low frequency and complex features. Grade 3 is composed of two subgroups, one with high ventricular extrasystoles frequency and another with low frequency. Grade $4 \mathrm{~A}$ is composed of four subgroups depending on the presence or absence of high ventricular extrasystoles frequency and multiform ventricular extrasystoles. Grade 4B is composed of eight subgroups depending on the presence or absence of high frequency of ventricular extrasystoles, multiform ventricular extrasystoles, and pairs of ventricular extrasystoles. Grade 5 is composed of 16 subgroups depending on the presence or absence of high frequency ventricular extrasystoles, multiform ventricular extrasystoles, ventricular extrasystoles pairs, and ventricular tachycardia. Feinstein has pointed out that, for prognostic stratification, subgroups should not be aggregated unless they are homogeneous with respect to outcome. ${ }^{9}$ Therefore, we examined the homogeneity of the subgroups in grades 3 to 5 with respect to death during follow-up. Only grade 5 had enough patients in several subgroups to make such comparisons useful (see Table 5). Three of the possible subgroups in grade 5 did not occur in our sample. The death rate among grade 5 subgroups ranged from 0 to 75 per cent. Two $a$ priori contrasts were made: (1) between those patients with only $R$ on $T$ ventricular extrasystoles and those with all complex ventricular extrasystoles features and (2) between those patients who had repetitive ventricular extrasystoles forms and those who did not. The 22 patients who had low ventricular extrasystoles frequency and no complex feature except the $R$ on $T$ phenomenon had a 9 per cent mortality, far below the overall 29 per cent death rate in grade 5 . There were 17 patients who had high ventricular extrasystoles frequency and all complex features; the death rate in this group was 59 per cent. The difference between these two subgroups was highly significant $(z=3.00, p<0.001)$. In addition, we compared the mortality rates of patients in grade 5 who had repetitive ventricular extrasystoles with those who did not. The 55 patients who had paired ventricular extrasystoles 
had a 49 per cent mortality rate compared with a 12 per cent mortality in the 61 patients who did not $(\mathrm{z}=4.19, \mathrm{p}<0.001)$. The 26 patients who had ventricular tachycardia had a 58 per cent mortality rate compared with a 21 per cent mortality in the 90 patients who did not $(z=3.36, p<0.001)$. The 58 patients in grade 5 who had repetitive ventricular extrasystoles, either paired ventricular extrasystoles or ventricular tachycardia, had a 48 per cent mortality rate compared with a 10 per cent mortality in the 58 patients who did not $(z=4 \cdot 26, p<0.001)$. In view of these findings, we concluded that there is very significant heterogeneity among the subgroups in Lown grade 5 . High and low risk subgroups are admixed in grade 5 ; furthermore, the high and low risk groups can be separated easily using results of 24-hour electrocardiographic recordings. The presence or absence of high ventricular extrasystoles frequency and/or repetitive ventricular extrasystoles are the most important features for this purpose.

\section{Discussion}

Although the mere presence of ventricular extrasystoles does effectively stratify risk, it lacks specificity. ${ }^{11}$ Lown believes that the ubiquity of ventricular extrasystoles militates against their use in identifying patients with ischaemic heart disease who are at high risk of death. ${ }^{15} \mathrm{He}$ has suggested that certain ventricular extrasystoles characteristics are harbingers of sudden death. ${ }^{15}$ The Lown grading system for ventricular arrhythmias embodies this hypothesis into a hierarchy of features that can be used to partition patients with ischaemic heart disease into grades of ever increasing risk of cardiac death. ${ }^{16}$ Our data indicate that Lown's fundamental hypothesis is correct, that is frequency and the four ventricular extrasystoles characteristics used in the Lown grading system can stratify the risk of patients with ischaemic heart disease. Our study suggests, however, that the Lown method of grading ventricular arrhythmias has several flaws when used for prognostic stratification in patients with ischaemic heart disease. Failures of the Lown grading system to predict outcome in ischaemic heart disease stem from its hierarchial and mutally exclusive use of ventricular extrasystoles features. The Lown grading system underuses information about frequency of ventricular extrasystoles. Only one partition value ( $\geq 30$ per hour) is used for patients who have ventricular extrasystoles. Our data show an increase in likelihood of death as ventricular extrasystoles frequency increases (see Fig. 1). In addition, the Lown grading system does not use ventricular extrasystoles frequency in risk stratification in the presence of complex ventricular extrasystoles features. This practice results from the assumption that the risk force of ventricular extrasystoles frequency is negligible in the presence of complex ventricular extrasystoles, that is that complex ventricular extrasystoles are more potent risk factors. In our study, this assumption was proved false. In Lown grades 4 to 5 , the additional use of ventricular extrasystole frequency significantly improved the risk stratification. Further, the hierarchy of complex features is flawed. Grade 5 should have the highest risk but the mortality rate in grade 5 is not significantly different from that in grades $4 \mathrm{~A}$ or $4 \mathrm{~B}$.

Another flaw in the higher Lown grades is the heterogeneity of outcome among the subgroups that make up a grade. This inadequacy was particularly striking in grade 5 where very high and very low risk subgroups can be identified. The patients in grade 5 with low ventricular extrasystoles frequency and no complex feature except $R$ on $T$ have a very low mortality rate $(9 \%)$ whereas those with a high frequency and all complex features have a very high mortality rate $(59 \%)$. The admixture of low and high risk subgroups into a single "high risk" grade has the potential for causing harm to low risk patients in intervention trials. Low risk patients could inadvertently and unnecessarily be exposed to treatments which carry significant potential for adverse effects. In addition, misclassification of very low risk patients into "high risk" groups can increase the costs of intervention trials. Larger sample sizes will be needed to compensate for higher misclassification rates. ${ }^{8}$

The best predictors of death in our study were ventricular extrasystoles frequency and repetitive ones, that is either paired ventricular extrasystoles or ventricular tachycardia. Lown also has been impressed with the prognostic significance of repetitive ventricular extrasystoles. ${ }^{56}$ Our analysis indicates that their frequency and the repetitive forms can be used together to identify the ischaemic heart disease groups with very high risk of subsequent death. Our study also suggests the hypothesis that significant reduction in frequency of ventricular extrasystoles and, particularly, in repetitive ventricular extrasystoles would reduce the incidence of subsequent cardiac death. This hypothesis may or may not be true, but it is both important and straightforward to test. There are several trial designs that could be used to test this hypothesis; a full discussion of these is beyond the scope of this communication. One approach, however, would be to use multiple doses and/or drugs guided by electrocardiographic analysis to determine whether or not frequency and repetitive ventricular extrasystoles are actually reduced to the target levels; 
the outcome could then be related to the success or failure of drug treatment. An exciting pilot study by Graboys et al. has incorporated many of these principles and provides support for the hypothesis. ${ }^{12}$

Supported in part by a grant-in-aid from the American Heart Association, by an NIH grant, and by grants-in-aid from the Winthrop Foundation and the Chernow Foundation.

\section{References}

1 Lown B, Wolf M. Approaches to sudden death from coronary heart disease. Circulation 1971 ; 44: 130-42.

2 Calvert A, Lown B, Gorlin, R. Ventricular premature beats and anatomically defined coronary heart disease. Am $\mathcal{f}$ Cardiol 1977; 39: 627-34.

3 Schulze RA, Humphries JO, Griffith LSC, et al. Left ventricular and coronary angiographic anatomy: relationship to ventricular irritability in the late hospital phase of acute myocardial infarction. Circulation 1977; 55: 839-43.

4 Schulze RA Jr, Strauss HW, Pitt B. Sudden death in the year following myocardial infarction: relation to ventricular premature contractions in the late hospital phase and left ventricular ejection fraction. Am f Med 1977; 62: 192-9.

5 Lown B, Calvert AF, Armington R, Ryan M.
Monitoring for serious arrhythmias and high risk of sudden death. Circulation 1975; 51 \& 52, suppl 3: 189-98.

6 Lown B, Graboys TB. Management of patients with malignant ventricular arrhythmias. Am $\mathcal{f}$ Cardiol 1977; 39: 910-8.

7 Birman KP, Rolnitzky LM, Bigger JT Jr. A shape oriented system for automated Holter ECG analysis. In: Computers in cardiology. Long Beach, California: IEEE Computer Society, 1974: 217-20.

8 Fleiss JL. Statistical methods for rates and proportions. New York: John Wiley, 1973.

9 Feinstein AR. Clinical biostatistics. St Louis: CV Mosby, 1977.

10 Bigger JT Jr. New directions and new uses for risk stratification in the post-hospital phase of acute myocardial infarction. $\mathrm{Am} \mathcal{F}$ Med 1979; 67: 1-2.

11 Moss AJ, Davis HT, DeCamilla J, Bayer LW. Ventricular ectopic beats and their relation to sudden and nonsudden cardiac death after myocardial infarction. Circulation 1979; 60: 998-1003.

12 Graboys TB, Lown B, Podrid PJ, DeSilva RA. Survival of patients with malignant ventricular arrhythmia treated with antiarrhythmic agents (abstract). Circulation 1979; 60, suppl 2: 255.

Requests for reprints to Dr J T Bigger Jr, Department of Medicine, Columbia University, 630 West 168th Street, New York, New York 10032, USA. 